\title{
AN INHERENT NONLINEARITY IN NEAR-THRESHOLD CONTRAST DETECTION
}

\author{
DAN J. SWIFT ${ }^{1.2}$ and ROBERT A. SMITH' \\ 'Vision Research Laboratory, University of New Hampshire. Pettee Brook Offices, Durham, NH 03824 \\ and 'Department of Behavioral Sciences, University of Michigan, Dearborn, MI 48I28, U.S.A.
}

(Receited 6 September 1983: in retised form 30 January 1984)

\begin{abstract}
We measured an essentially normal pedestal effect using stationary gaussian targets and slowly moving pedestal gratings. Since these conditions greatly reduce the information provided by the pedestal, we question whether uncertainty about the stimulus can be the main cause of the pedestal effect.
\end{abstract}

\section{INTRODUCTION}

In 1974 Nachmias and Sansbury studied the detection of gratings at low contrast when superimposed upon a near-threshold pedestal grating of the same frequency and phase. They found that low pedestal contrasts actually facilitate detection of the test grating. The effect is significant for pedestal contrasts within about a factor of two of threshold, and for pedestal frequencies within about half an octave of the test frequency (Legge and Foley, 1980). This phenomenon has been widely replicated (Stromeyer and Klein, 1974; Smith and Swift, 1978; Legge and Foley, 1980). Nachmias and Kocher (1970) reported a similar phenomenon with unpatterned spots of light, and offered two hypotheses: (1) the visual system may perform a positively-accelerated, nonlinear transformation on low-contrast signals. For example, Legge and Foley (1980) modeled this effect with a power law (exponent $=2.8$ ); (2) The observer may use the pedestal as a cue to reduce stimulus uncertainty, especially positional uncertainty, thereby facilitating detection.

We propose to test these hypotheses with an experiment in which the pedestal provides little or no information about the test stimulus. If this eliminates facilitation, we have good evidence for an uncertainty explanation. Conversely, if facilitation is unaffected, then the case for an inherent nonlinearity is strengthened.

\section{METHODS}

The stimuli used in this experiment were produced on an HP 1332A CRT by conventional means. The CRT was white (P4 phosphor), had a background luminance of $50 \mathrm{~cd} / \mathrm{m}^{2}$, and was $8^{\circ}$ high by $10^{\circ}$ wide. All stimuli extended the full screen height. In the absence of test stimuli, the screen was dark (about $7 \mathrm{~cd} / \mathrm{m}^{2}$ from room illumination). The raster contained 700 vertical lines, displayed at a rate of $80 \mathrm{frames} / \mathrm{sec}$. The test and pedestal stimuli were superimposed by displaying them on alternate frames. Stimuli were produced and selected by a microcomputer which also recorded the subject's responses.

Test stimuli were vertical gaussian bars, 2 standard deviations wide. These were not truncated but were brought smoothly to zero luminance by a linear ramp $1 / 2$ standard deviation wide adjoined to each edge of the Gaussian. The subject (an experienced psychophysicist) carefully fixated a spot in the center of the screen and test stimuli were presented $11 / 2 \mathrm{deg}$ to either side of that spot, using a two-alternative forced-choice staircase described in Swift and Smith (1982). The test stimulus was present indefinitely, until the observer decided whether it was to the right or left of the fixation spot. Pilot experiments showed the standard error of all thresholds to be under $15 \%$, requiring 30-40 trials. To reduce the observer's uncertainty, each bar width was run separately. The superimposed pedestal stimuli were sine-wave gratings of varing frequency, moving at $1.0 \mathrm{deg} / \mathrm{sec}$, and covering the entire screen. Test intensity was measured in percent, where $100 \%$ was a pattern whose brightest point was twice the mean luminance; pedestal contrast had the conventional Michaelson definition.

\section{RESULTS AND DISCUSSION}

Figure 1 shows detection thresholds for the test bar as a functional of pedestal contrast for three bar sizes. Facilitation is greastest for a pedestal contrast of about $0.75 \%$, which is slightly above the pedestal's threshold. Facilitation is considerably greater for the $0.9 \mathrm{deg}$ wide bar. This apparent size-tuning is explored further in a second experiment (Fig. 2), studying the amount of facilitation vs test bar width for two pedestal frequencies. Facilitation is clearly tuned for the relative size of the pedestal and test bar, being maximum when the bar width approximately equals the half-wavelength of the pedestal.

The pedestal effect with gaussian targets is similar to that reported with sinusoidal targets (see Introduction). The peak amount of facilitation is com- 


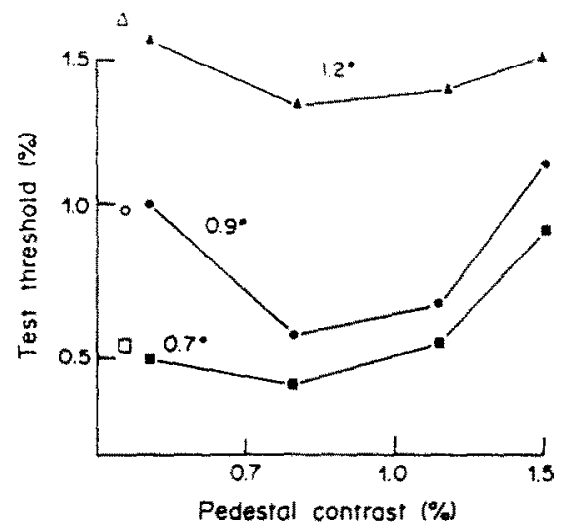

Fig. 1. Detection thresholds for three sizes of test bar, as a function of pedestal contrast. Open symbols show thresholds without the pedestal. Pedestal was $0.5 \mathrm{c} / \mathrm{deg}$. moving $1.0 \mathrm{deg} / \mathrm{sec}$. Semi-log coordinates; standard error is $15 \%$.

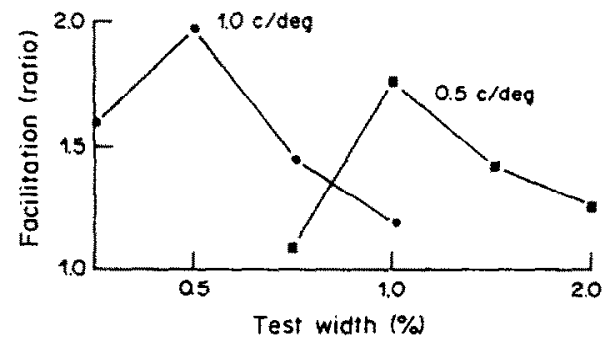

Fig. 2. Amount of facilitation (expressed as the ratio of unfacilitated to facilitated threshold) vs test bar width, for two frequencies of pedestal. Pedestal contrast was 0.85 , moving $1.0 \mathrm{deg} / \mathrm{sec}$. Semi-log coordinates

parable, and in both cases this occurs when test and pedestal are of about the same shape. It is a little surprising that enhancement falls off at lower, as well as higher, pedestal frequencies, since the spectrum of a Gaussian bar does not fall off at low frequencies. This apparent tuning may be artifactual, since the observer's sensitivity falls rapidly at low frequencies. Thus even if low frequencies are facilitated, this may not improve detection.

Facilitation is probably not due to reduced uncertainty, for several reasons. (1) The test stimulus was present continually, so temporal uncertainty is not a factor. (2) Since the stimuli were blurred-edge bars, on the order of 1 deg wide, precise spatial localization seems superfluous: placement of the stimuli $1.5 \mathrm{deg}$ from the fixation point provides considerable information about stimulus position. (3) A continuously moving pedestal provides no information at all about stimulus position. (4) Since stimuli of different widths were not intermixed in a given staircase, it is unlikely there was signiticant uncertainly about the size of the stimulus, though we acknowledge that the observed size tuning suggests a size-uncertainty explanation. In conclusion, finding an essentially normal pedestal effect in the near-absence of any uncertainty, we conclude that some other form of threshold nonlinearity is probably present.

We did not carefully investigate the effect of pedestal velocity, but this must be quite slow. At higher velocities the pedestal becomes much more visible, and actually masks the test. Our data suggest that slow velocity does not substantially change the facilitation effect from that described by Nachmias and Kocher; rather the motion merely removes positional information from the pedestal. It is unlikely that the moving pedestal significantly affects eye movements; Murphy et al. (1975) found fixation to be almost totally resistant to the effect of a moving grating, even at high contrast. In any case, it seems unlikely that eye movements could produce our specific results.

We are not arguing against uncertainty as a sometime cause of the pedestal effect. The a priori reasons for postulating such an effect seem to us very convincing, as do some experimental demonstrations (e.g. Lasley et al., 1983). In fact, the two explanations are by no means incompatible, and we believe that both may occur under appropriate conditions.

Acknowledgement-This work was supported by AFOSR Grant No. 80-0045.

\section{REFERENCES}

Foley J. M. and Legge G. E. (1981) Contrast detection and near-threshold discrimination in human vision. Vision Res. 21, 1041-1053.

Lasley D. J., Silverman C. A. and Cohn T. E. (1983) Site of the accelerating nonlinearity for detection. Invest Ophthal. visual Sci., Suppl. No. 3. 24, 144.

Legge G. E. and Foley J. M. (1980) Contrast masking in human vision. J. opt. Soc. Am. 70, 1458-1471.

Murphy B. J., Kowler E. and Steinman R. Slow oculomotor control in the presence of moving backgrounds. Vision Res. 15, 1263-1268.

Nachmias J. and Kocher E. C. (1970) Visual detection and discrimination of luminance increments. J. opt. Soc. Am. 60, 382-389.

Nachmias J. and Sansbury R. V. (1974) Grating contrast discrimination may be better than detection. Vision Res. 14, 1039-1042.

Smith R. A. and Swift D. J. (1978) Simultaneous masking and the bandpass of spatial-frequency channels. Presented at $A R V O$, Sarasota, Florida (1978).

Swift D. J. and Smith R. A. (1982) An action spectrum for spatial-frequency adaptation. Vision Res. 22, 235-246.

Stromeyer C. F. III and Klein S. (1974) Spatial frequency channels in human vision as asymmetric (edge) mechanisms. Vision Res. 14, 1409-1420. 